\title{
Responses of Monkey Dopamine Neurons to Reward and Conditioned Stimuli during Successive Steps of Learning a Delayed Response Task
}

\author{
Wolfram Schultz, Paul Apicella, ${ }^{a}$ and Tomas Ljungberg ${ }^{b}$ \\ Institut de Physiologie, Université de Fribourg, $\mathrm{CH}-1700$ Fribourg, Switzerland
}

The present investigation had two aims: (1) to study responses of dopamine neurons to stimuli with attentional and motivational significance during several steps of learning a behavioral task, and (2) to study the activity of dopamine neurons during the performance of cognitive tasks known to be impaired after lesions of these neurons. Monkeys that had previously learned a simple reaction time task were trained to perform a spatial delayed response task via two intermediate tasks. During the learning of each new task, a total of $25 \%$ of 76 dopamine neurons showed phasic responses to the delivery of primary liquid reward, whereas only $9 \%$ of 163 neurons responded to this event once task performance was established. This produced an average population response during but not after learning of each task. Reward responses during learning were significantly more numerous and pronounced in area $A 10$, as compared to areas A8 and A9. Dopamine neurons also showed phasic responses to the two conditioned stimuli. These were the instruction cue, which was the first stimulus in each trial and indicated the target of the upcoming arm movement $(58 \%$ of 76 neurons during and $44 \%$ of 163 neurons after learning), and the trigger stimulus, which was a conditioned incentive stimulus predicting reward and eliciting a saccadic eye movement and an arm reaching movement (38\% of neurons during and $\mathbf{4 0} \%$ after learning). None of the dopamine neurons showed sustained activity in the delay between the instruction and trigger stimuli that would resemble the activity of neurons in dopamine terminal areas, such as the striatum and frontal cortex. Thus, dopamine neurons respond phasically to alerting external stimuli with behavioral significance whose detection is crucial for learning and performing delayed response tasks. The lack of sustained activity suggests that dopamine neurons do not encode representational processes, such as working memory, expectation of external stimuli or reward, or preparation of movement. Rather, dopamine neurons are involved with transient changes of impulse activity in basic attentional and

\footnotetext{
Received June 17, 1992; revised Sept. 3, 1992; accepted Sept. 9, 1992.

We thank Dr. J. R. Hollerman for comments on the manuscript and F. Tinguely, A. Schwarz, and J. Corpataux for technical assistance. The study was supported by the Swiss NSF (Grants 3.473-0.86, 31-28591.90) and the United Parkinson Foundation. Postdoctoral fellowships to P.A. were provided by the Fyssen Foundation and the Fondation pour la Recherche Médicale.

Correspondence should be addressed to Dr. W. Schultz at the above address.

aPresent address: Laboratory of Cellular and Functional Neurobiology, CNRS, F-13274 Marseille, France.

bPresent address: Department of Pharmacology, Karolinska Institute, S-10401 Stockholm, Sweden.

Copyright (C 1993 Society for Neuroscience $0270-6474 / 93 / 130900-14 \$ 05.00 / 0$
}

motivational processes underlying learning and cognitive behavior.

[Key words: behavior, motivation, learning, conditioning, reward, cognition, attention]

In previous neurophysiological studies in behaving monkeys, it was found that dopamine (DA) neurons respond to primary food and fluid rewards and to conditioned incentive stimuli predicting reward (Schultz, 1986; Romo and Schult7, 1990; Ljungberg et al., 1992). Responses to conditioned stimuli have also been observed in awake, haloperidol-treated rats (Miller et al., 1981). DA neurons also respond to novel, unexpected stimuli, both in cats (Steinfels et al., 1983) and in monkeys (Ljungberg et al., 1992). These data suggest that DA neurons respond to salient environmental stimuli that alert the animal and attract its attention. Large, albeit not exclusive, classes of these effective stimuli are primary and learned appetitive motivating stimuli.

Primary rewards, novel stimuli, and conditioned incentive stimuli play particularly prominent roles when an animal learns new or changed behavioral contingencies for obtaining reward. We have investigated the responses of DA neurons to various stimuli while animals learned simple reaction time tasks (Ljungbcrg ct al., 1992). It was found that neurons responded to the sight or reception of primary reward during learning, and that these responses were transferred to the conditioned stimulus predicting reward when task performance was established. These responses to the conditioned stimulus were strongly reduced after overtraining. These data suggest that the responses of DA neurons may be particularly important during learning when the animal adapts its behavior to new situations.

The present study aimed to answer two questions. (1) If the responses of DA neurons are transferred between the most significant stimuli during the course of learning, then how would these neurons respond when the learning of a more elaborate task requires a consecutive series of intermediate subtasks and does not occur in basically a single step, as with a reaction time task? More complicated tasks performed by monkeys in a laboratory setting could comprise the choice of different spatial targets for the behavioral response and involve working memory and the preparation of movement. (2) It would be particularly interesting to see how the activity of DA neurons changes during the performance of such tasks, since Parkinsonian patients and animals with lesions of DA neurons show considerable difficulties in performing tasks requiring the use of declarative cognitive or procedural representations (Brozoski et al., 1979; Simon et al., 1980; Cools et al., 1984; Saint-Cyr et al., 1988; Schneider and Kovelowski, 1990). In combining these two questions, we 

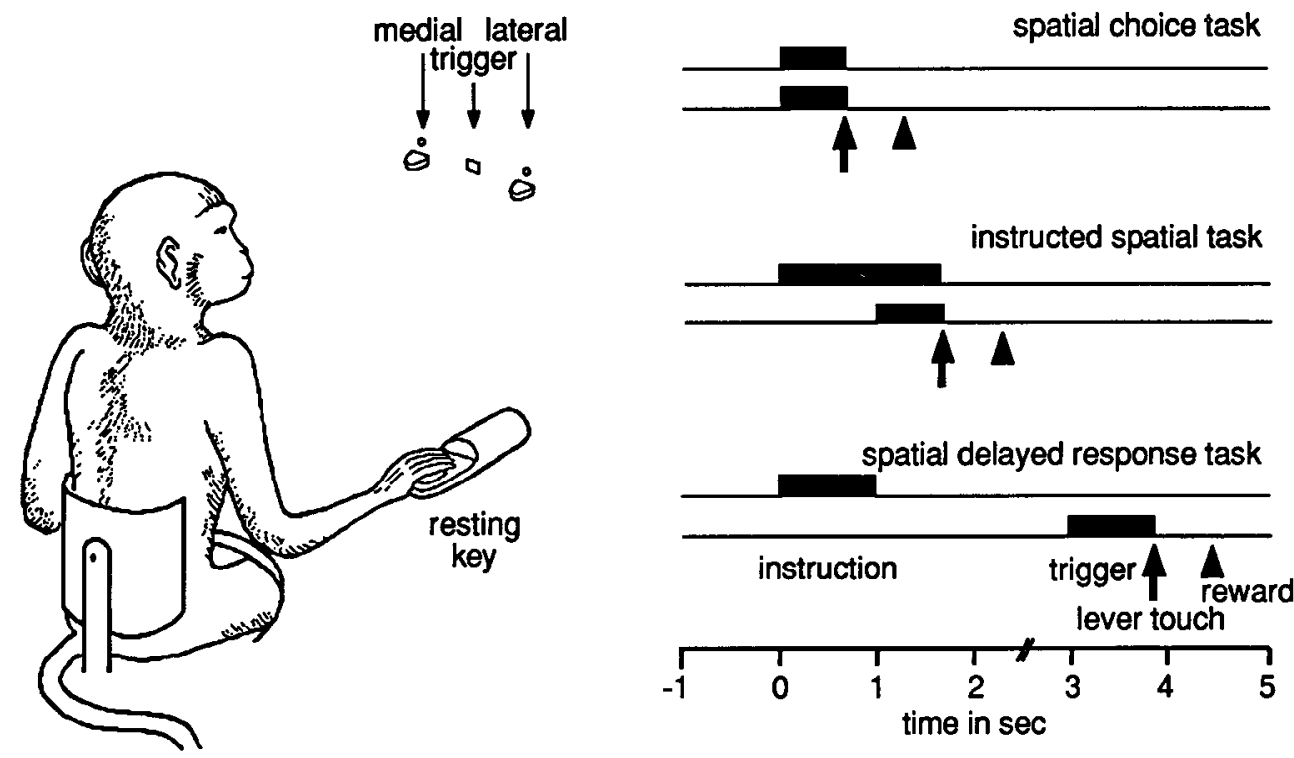

Figure 1. Schematic description of behavioral tasks. A trial is initiated when the animal keeps its hand relaxed on the immovable resting key. Illumination of an instruction light above the medial or lateral lever indicates the future target of reaching. The trigger light determines the time when the resting key should be released and the lever indicated by the instruction touched. Medial and lateral levers are used in random alternation. In the spatial choice task, both instruction and trigger lights come up at the same time and are extinguished upon lever touch. In the instructed spatial task, the trigger light comes up 1 sec after the instruction, and both lights are extinguished upon lever touch. In the spatial dclaycd response task, the trigger comes up after a randomly varying interval of 2.5-3.5 sec after instruction onset. The instruction is extinguished 1 sec after it came up. Thus, the animal cannot precisely predict when the trigger light comes up and needs to retain the spatial position of the lever until it is allowed to move in response to the trigger. Reward is given in all tasks $0.5 \mathrm{sec}$ after correct lever touch.

studied DA neurons while monkeys learned in several steps a spatial delayed response task in which they needed to remember the spatial position of a cue for several seconds, prepared for an upcoming arm movement, and executed it when a trigger stimulus came up. Because we only wanted the animals to advance in learning the tasks while we recorded from a DA neuron, task acquisition was facilitated by using animals that had already learned a simple reaction time task (Ljungberg et al., 1992), and by employing the most basic version of a delayed response task with only two reaching targets that were constantly visible to the animals.

These data have been presented previously in short form (Schultz et al., 1991).

\section{Materials and Methods}

The study was performed on two male Macaca fascicularis monkeys of $3.5 \mathrm{~kg}$ weight. Activity of single neurons was recorded with moveable microelectrodes during learning and established performance of three consecutive spatial reaching tasks. Animals were killed for histological reconstruction of recording sites. A third monkey was used before the recording experiments for the development of task learning. The behavioral apparatus and recording techniques were similar to those previously reported (Schultz, 1986; Ljungberg et al., 1992).

\section{Behavioral procedures}

The behavioral apparatus was positioned at reaching distance $(250 \mathrm{~mm}$ from the animal's shoulder) in the right half of the frontal wall of a completely enclosed primate chair (Fig. 1). A yellow, rectangular lightemitting diode $(11 \times 11 \mathrm{~mm})$ was mounted at $27^{\circ}$ lateral to the midsagittal plane and at eye level of the animal. Two small levers $(7 \times 15$ $\mathrm{mm}$ ) were placed $40 \mathrm{~mm}$ medial and lateral to the yellow light. Levers protruded by $20 \mathrm{~mm}$ from the frontal wall and made electrical contact upon downward movement of $1 \mathrm{~mm}$. One green, round light-emitting diode of $3 \mathrm{~mm}$ diameter was located $10 \mathrm{~mm}$ above each lever. An immovable, touch-sensitive key was mounted below the lights at waist level such that the elbow joint of the animal was kept at approximately $90^{\circ}$ when its hand rested on the key. A drop of apple juice $(0.15-0.20$ $\mathrm{ml}$ ) served as reward, which was delivered by an electronically controlled solenoid valve. The solenoid emitted an audible click upon complete opening at $18 \mathrm{msec}$ after the electronic pulse. Liquid arrived at the spout in front of the animal's mouth with an average delay of $55 \mathrm{msec}$ after the electronic pulse. Two closed-circuit video systems served to supervise limb and mouth movements continuously. Monkeys were deprived of fluid during weekdays. They were released into their home cages after each daily experiment of $3-4 \mathrm{hr}$ and received water ad libitum during the subsequent $1 \mathrm{hr}$.

Both animals had been conditioned to perform in a simple reaction time task (Ljungberg et al., 1992) in which they kept their one hand relaxed on the resting key until the yellow light came on as trigger stimulus. The animal released the key in response to this stimulus, reached out, and depressed the small lever below the light for liquid reward. In the present tasks, a spatial component of reaching was introduced by using two target levers instead of one. The correct lever was indicated by the instruction light shown at different times in the three tasks.

Spatial choice task. The instruction light situated above one of the levers came up at the same time as the central trigger light. The animal released the key in response to the two simultaneous lights, reached out, and depressed the lever indicated by the instruction light in order to receive the reward. Both lights were extinguished upon lever touch or, if no movement occurred, after $1 \mathrm{sec}$. Liquid reward was delivered 500 msec after correct lever touch. This delay allowed the separation of neuronal responses to lever touch from those to reward. Trials lasted 6-9 sec; intertrial intervals varied between 3 and $5 \mathrm{sec}$. The use of the lateral and medial levers alternated randomly between trials, but the successive use of the same lever was limited to maximal three trials.

Instructed spatial task. The instruction light situated above one of the levers came up at a fixed interval of $1 \mathrm{sec}$ before the central trigger light. The animal released the resting key and touched the lever in response to the trigger light. Thus, the instruction light indicated the lever to be touched and served as preparatory signal for the upcoming behavioral response, whereas the trigger light determined the time of responding without providing spatial information. Both lights were extinguished upon lever touch or, if no movement occurred, after $1 \mathrm{sec}$. Reward was delivered $500 \mathrm{msec}$ after correct lever touch.

Spatial delayed response task. The instruction light indicating the lever to be touched came up for a fixed duration of 1 sec. The central light triggering the reaching movement was illuminated at a randomly varied interval of 2.5-3.5 sec after instruction onset and was extinguished upon 
lever touch or, if no movement occurred, after $1 \mathrm{sec}$. Reward was delivered $500 \mathrm{msec}$ after touch of the corrcet lcver. This situation constitutes a spatial delayed response task in which the animal has to remember the lever to be touched.

Learning of tasks. The animals, previously trained in the simple reaction time task, were first conditioned to perform the spatial choice task. After reaching stable performance for about 1 week, the task was abandoned and the instructed spatial task was introduced. After stable performance for about 1 week, this task was in turn abandoned for the spatial delayed response task. During conditioning in the spatial choice and the instructed spatial tasks, the new contingencies were introduced in one step and animals had to find the correct solution to the new problem by their own trial and error attempts. Learning of the spatial delayed response task involved a stepwise approach to the longer interval and delay. For monkey $A$, intervals between onsets of instruction and trigger stimuli were gradually prolonged to $3 \mathrm{sec}$, and the duration of the instruction cue was subsequently shortened to $1 \mathrm{sec}$, thus introducing the delay. For monkey $\mathrm{B}$, the duration of the instruction cue was kept at $\mathbf{l ~ s e c}$, and intervals between instruction and trigger stimuli were gradually prolonged. The acceptable interval for lever pressing after the trigger stimulus was initially set to $3 \mathrm{sec}$ and gradually shortened to the final 1 sec.

Learning of each task was best described by the frequency of correct responses. Systematic changes in arm reaction time and movement time were not observed during learning, possibly because of extensive training in the previous reaction time task involving a comparable arm movement. Thus, each task was divided into the learning and the established performance parts according to the time when a stable frequency of correct responses was reached.

\section{Data acquisition}

All bchavioral performance was controlled by a suitably interfaced laboratory computer, the selection of appropriate task parameters being done by the experimenters individually for each block of trials. The different lights and the solenoid for reward delivery were driven by output pulses from the computer. Key release was detected by a frequency-sensing circuit that reacted to a change in electrical capacity induced by the touch of the animal's hand. Errors in behavioral performance led either to cancellation of all further signals in a given trial, including reward, or to immediate trial restart.

Animals had been implanted for the preceding study on reaction time tasks. Under deep sodium pentobarbital anesthesia and aseptic conditions, cylinders for head fixation and a sterentaxically positioned, stainless steel chamber were fixed to the skull to permit vertical access with microelectrodes to the left substantia nigra. The dura was left intact. Tefion-coated, multistranded, stainless steel wires were implanted into the extensor digitorum communis and biceps muscles of the arm and led subcutaneously to the head. $\mathrm{Ag}-\mathrm{AgCl}$ electrodes were implanted into the outer, upper, and lower canthi of the orbits. All metal components, including connector plugs for the muscle and periorbital electrodes, were embedded in several layers of dental cement and fixed to the skull with surgical grade stainless steel screws. One week after implantation, animals were anesthetized with pentobarbital and the area of substantia nigra was localized by taking lateral and coronal radiographs with a guide cannula installed at a known coordinate in reference to the implanted steel chamber. The ventroposteromedial thalamus overlying the lateral substantia nigra was electrophysiologically explored for trigeminal input on the same occasion, and later occasionally in the waking animal.

The activity of single neurons was recorded extracellularly with glassinsulated, platinum-plated tungsten microelectrodes (exposed tips of 9$16 \mu \mathrm{m}$ length and 2.5-3.5 $\mu \mathrm{m}$ diameter), which were passed each day into the brain, together with and inside of a rigid guide cannula of 0.6 $\mathrm{mm}$ outer diameter. Microelectrodes were moved vertically in the stereotaxic plane in parallel tracks that conformed to a $1 \mathrm{~mm}$ grid. Signals from the microelectrode were conventionally amplified, filtered (100 $\mathrm{Hz}$ lower cutoff), and monitored with oscilloscopes and audiomonitors using earphones to prevent the monkeys from hearing the neuronal activity. Full waveforms of impulses from each neuron were displayed on a digital oscilloscope using the pretrigger viewing facility and subsequently stored on computer disks. Somatodendritic discharges were discriminated against those originating from fibers using earlier established criteria, in particular the very short durations of fiber impulses (0.1-0.3 msec) (Hellweg et al., 1977; Schultz and Romo, 1987). Neuronal discharges were also converted into standard digital pulses by means of an adjustable Schmitt trigger, the output of which was continuously monitored on the digital oscilloscope together with the original waveform. DA neurons were searched for in a systematic fashion. Every neuron fulfilling the criteria for being dopaminergic was tested with at least 20 trials of a given task situation and included in the study.

Electromyograms (EMGs) were collected during all neuronal recordings through the chronically implanted electrodes. EMG activity was filtered $(10-250 \mathrm{~Hz}$ bandpass; $-12 \mathrm{~dB}$ at $1 \mathrm{kHz})$, rectified, and displayed on conventional oscilloscopes. Rectified EMG activity was also passed through an adjustable Schmitt trigger and fed to the computer. Horizontal and vertical electrooculograms (EOGs) were collected during all neuronal recordings from the implanted periorbital electrodes. The gain of ocular electrodes and positions of the eyes were calibrated by having the animal fixate small morsels of preferred food presented at several known horizontal and vertical eccentricities while the frontal enclosure of the primate chair was kept open. Direct current offset had to be adjusted once every $3-4$ weeks.

All behavior-related digital signals and pulses from neuronal discharges and EMG activity were sampled on line as bits in parallel at a rate of $2 \mathrm{kHz}$ by a laboratory computer. Analog signals from EOGs and rectified EMGs were sampled after analog-to-digital conversion at a rate of $2 \mathrm{kHz}$ by the computer. Eight consecutive analog values were averaged to obtain a final temporal resolution of $4 \mathrm{msec}(0.25 \mathrm{kHz})$ for data storage. The behavioral relationships of neuronal discharges, digital and analog EMG activity, and EOGs were displayed in each trial on line on the computer video screen in the form of dot displays and analog curves. All data were stored uncondensed on computer disks.

\section{Data analysis}

Because of the stereotyped character of phasic responses of DA neurons to external stimuli seen before (Schultz, 1986; Romo and Schultz, 1990; Schultz and Romo, 1990), a standard time window procedure was developed in order to quantify and compare objectively the neuronal responses between different parts of experimentation (Ljungberg et al., 1992). First, onset and offset times of all phasic activations in response to the conditioned stimuli and delivery of liquid reward were determined with a resolution of $4 \mathrm{msec}$ from the first or last of at least three consecutive bins in which activity was above or back to baseline, respectively, as indicated by dot displays, perievent time histograms, and the slopes of cumulative frequency distributions of neuronal impulses. The significance of an increase in activity over baseline was assessed with a specially implemented procedure using the two-tailed Wilcoxon matchedpairs signed-rank test $(p<0.01$; Schultz, 1986). Depressant responses to stimuli were tested for statistical significance of change in the standard time window, whereas onset and offset times were not measured in view of the low baseline activity of DA neurons.

For subsequent analysis, the standard time window was set to include $80 \%$ of onset and offset times of statistically significant increases in activity. Obtained time windows were $88-200$ msec after onsets of instruction and trigger lights and $172-256 \mathrm{msec}$ after delivery of liquid reward. Two measures for the responsiveness of neurons were sought. (1) The number of individual neurons showing a significant increase in activity in each time window was obtained with the Wilcoxon procedure. (2) The responsiveness of the whole population of neurons was assessed by measuring the magnitude of change of activity in the time windows against baseline activity for every neuron recorded in a particular task situation, independent of a significant response. For both measures, baseline activity was determined during the $500 \mathrm{msec}$ interval preceding presentation of the first stimulus of each trial. All neurons used for determining onset and offset times of significant increases in activity also showed significant activations in the standard time window. Magnitudes determined during the individual durations of response of each responding neuron were significantly higher by $20-30 \%$ than in the standard time windows for instruction, trigger, and reward used with the same neurons during both learning and established task performance in all three tasks $(p<0.001$, paired $t$ test).

In addition to the quantitative assessment of responses of individual neurons, the overall responsiveness of the entire population of neurons during particular tasks and learning phases was visually assessed from averages of neuronal activity. A peristimulus time histogram was constructed for every neuron recorded independent of a significant response, and the content of each of its bins was divided by the number of trials to obtain a normalized count. All normalized histograms referenced to the same behavioral event were averaged to obtain the population histogram. 


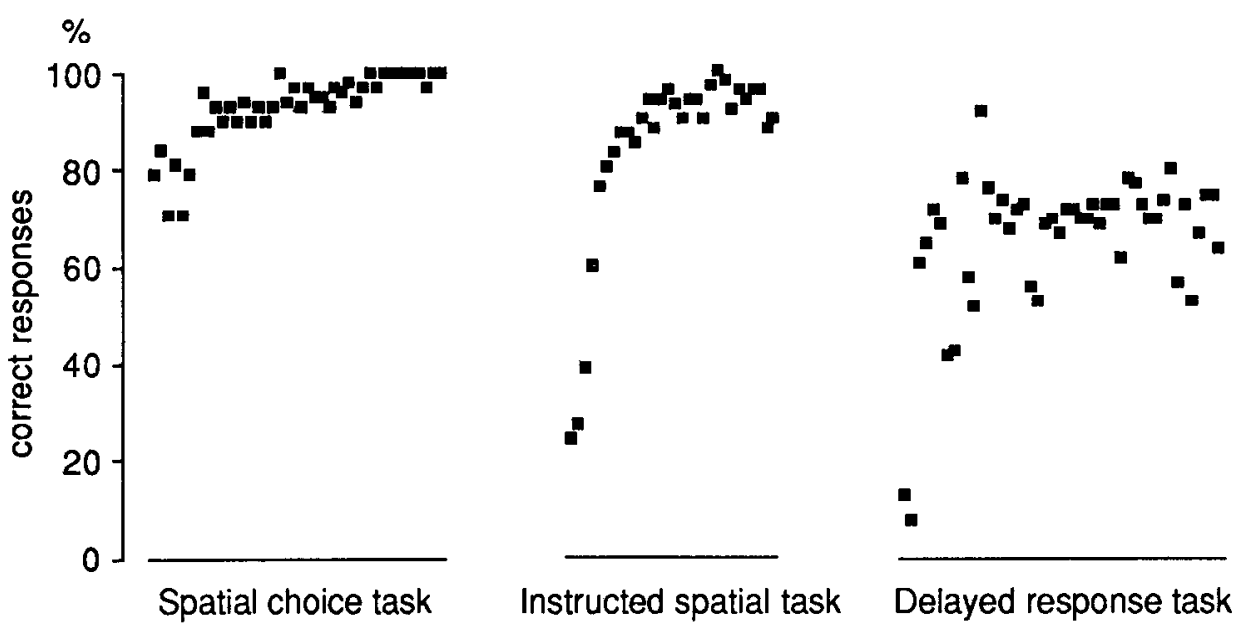

Figure 2. Learning curves for the three tasks in onc monkcy. Each data point was obtained during the recording of one DA neuron. Tasks were successively employed in the order shown from left to right.
Behavioral performance was assessed during the recording period of every neuron by calculating the median (50th percentile) of reaction time (from trigger light to key release) and movement time (from key release to lever touch) of correct behavioral responses.

Response magnitudes and medians of behavioral parameters were normally distributed (Kolmogorov-Smirnov one-sample test, $p>0.05$ ). Parametric statistics were therefore used, which included the means, standard errors of the mean (SEMs), and two-tailed Student's $t$ test for one sample and for paired and unpaired two samples. All timing and magnitude values are given as means \pm SEM. Correlations between magnitudes of changes after different stimuli were determined with Spearman's rank correlation coefficient. The $\chi^{2}$ test was employed for comparing the distributions of fractions of responding neurons among groups $\mathrm{A} 8, \mathrm{~A} 9$, and $\mathrm{A} 10$ against a hypothetically homogeneous distribution. Neuronal changes obtained from the standard time window method were compared with one-way and two-way ANOVAs using monkeys, phases of experimentation, or midbrain areas as factors. Data from neuronal activity were pooled over the two monkeys when systematic differences were absent.

\section{Histological reconstruction}

During the last recording sessions with each animal, small marking lesions were placed by passing negative currents $(10-20 \mu \mathrm{A}$ for $10-20$ sec) through the microelectrode immediately after recording from a neuron in the substantia nigra, while larger lesions $(20 \mu \mathrm{A}$ for 20 or 60 sec) were positioned at a few locations above in the same track. This produced distinct patterns of vertically oriented histological marks. Animals were deeply anesthetized with sodium pentobarbital and conventionally perfused with formaldehyde through the heart. Guide cannulas were inserted into the brain at known coordinates of the implant system in order to delineate the general area of recording. The tissue was cut in $50-\mu \mathrm{m}$-thick serial coronal sections on a cryotome and stained with cresyl violet. All histological sections were projected on paper, and the outlines of brain structures and the marks from lesions and recent electrode tracks were drawn. Recording positions in tracks marked by electrolytic lesions were reconstructed by using the distances to the lesions according to protocolled micrometer readings. Positions in parallel neighboring tracks were reconstructed at comparable vertical levels. The recorded DA neurons were attributed to groups A8, A9, and A10 according to previous histological descriptions of catecholamine cell groups in the monkey midbrain (Felten and Sladeck, 1983). Thus, group A9 comprised the pars compacta of substantia nigra, whereas group A8 was located dorsolaterally to the substantia nigra in the lateral reticular formation, and group A10 was found dorsomedially adjoining to and incompletely separated from the medial substantia nigra (lateral A10 region of Felten and Sladeck, 1983).

\section{Results}

General

Behavior. The gradual learning of each task is illustrated by the learning curves of Figure 2. Errors in the instructed spatial task consisted in precocious release of the resting key before the trigger stimulus, suggesting that animals used the instruction as stimulus for an appropriately delayed movement response. This was probably due to previous overtraining in the reaction time task in which animals moved in response to the first and only stimulus of each trial. In the spatial delayed response task, the interval between instruction and trigger stimuli was prolonged and varied to such an extent that animals only moved in response to the trigger light. Errors in this task mostly consisted in touching the wrong lever.

Performance in the spatial choice task levelled off at almost $100 \%$ correct responses, whereas it was successively lower in the two consecutive tasks of higher complexity. Learning of each task was considered to be terminated with a level of performance of $95 \%, 90 \%$, and $75 \%$ correct responses reached in the majority of blocks of trials in spatial choice, instructed spatial, and spatial delayed response tasks, respectively. During established task performance, reaction and movement times in the spatial choice and the instructed spatial tasks were in the same range as in the simple reaction time task employing the same movement (Ljungberg et al., 1992), whereas reaction times were considerably longer in the spatial delayed response task $(p<0.01$, Fisher test following one-way ANOVA) (Table 1).

Neurons. A total of 239 DA neurons were recorded in group A9 (pars compacta of substantia nigra, $n=162)$, A8 $(n=38)$, and A10 $(n=39)$. Neurons were recorded during learning and established performance of the spatial choice task, followed by recordings during learning and established performance of the instructed spatial task, and subsequently the spatial delayed response task. Histological reconstructions of recording sites revealed unsystematic, statistically insignificant differences in recording areas between tasks, and between learning and established performance of each task (Table 2).

As described before, midbrain DA neurons displayed specific electrophysiological characteristics (Schultz, 1986; Schultz and Romo, 1987). They discharged initially negative or positive impulses with low frequencies (0.5-9 impulses/sec), polyphasic waveforms, and relatively long durations (1.8-5.0 msec). They contrasted with pars reticulata neurons discharging impulses of $<1.1 \mathrm{msec}$ duration at rates of 70-90 impulses/sec, a few unknown neurons discharging impulses of $<1.0 \mathrm{msec}$ at low rates, and presumptive fibers discharging very short impulses (0.1$0.3 \mathrm{msec}$ ). 
Table 1. Behavioral performance after established task performance

\begin{tabular}{|c|c|c|c|}
\hline & $\begin{array}{l}\text { Reaction } \\
\text { time }(\mathrm{msec})\end{array}$ & $\begin{array}{l}\text { Movement } \\
\text { time (msec) }\end{array}$ & $\begin{array}{l}\text { Blocks of } \\
\text { trials } \\
(n)\end{array}$ \\
\hline \multicolumn{4}{|c|}{ Spatial choice task } \\
\hline Monkey A & $250 \pm 3^{1.2}$ & $349 \pm 4^{1,2}$ & 23 \\
\hline Monkey B & $271 \pm 3^{2}$ & $303 \pm 2^{2}$ & 32 \\
\hline \multicolumn{4}{|c|}{ Instructed spatial task } \\
\hline Monkey A & $224 \pm 7^{3}$ & $302 \pm 5$ & 19 \\
\hline Monkey B & $272 \pm 7^{3}$ & $312 \pm 3^{3}$ & 16 \\
\hline \multicolumn{4}{|c|}{ Spatial delaycd response task } \\
\hline Monkey A & $335 \pm 3$ & $310 \pm 3$ & 31 \\
\hline Monkey B & $346 \pm 7$ & $277 \pm 2$ & 42 \\
\hline
\end{tabular}

Values are given as means \pm SEM of performance in individual blocks of trials, each of which contained 40-64 trials. The differences between both monkeys and the three tasks were statistically significant $(p<0.001$, two-way ANOVA with monkeys and tasks as factors). Separately for each monkey, the following reaction and movement times varied significantly between the three tasks $(p<0.01$ in Fisher test after one-way ANOVA):

', spatial choice versus instructed spatial task;

${ }^{2}$, spatial choice versus spatial delayed response task;

${ }^{3}$, instructed spatial versus spatial delayed response task.

Data from movements to medial and lateral lever were pooled. All measures were obtained in trials in which DA neurons were recorded.

\section{Responses to reward}

Of 76 DA neurons recorded during the learning phases of the three tasks, 19 neurons (25\%) responded to the delivery of liquid reward. By contrast, after task performance was established only 14 of 163 neurons $(9 \%)$ were activated by reward (Fig. 3, Table 3 ). The activity of one neuron was depressed by reward. There were only insignificant differences in reward responses after reaching to the medial versus lateral levers. Of the total of 33 neurons responding to reward, 24 neurons $(73 \%)$ were also activated by the instruction cue.

The delayed delivery of reward after correct performance showed that reward responses occurred to the delivery of liquid and not to lever touch (Fig. 4, left). The reward response was equally present when reward was delivered simultaneously with correct lever touch. Nine neurons activated by reward in correct trials $(235 \pm 34 \%)$ were also recorded in $>10$ trials that were

\begin{tabular}{|c|c|c|}
\hline & $\begin{array}{l}\text { Task } \\
\text { learning }\end{array}$ & $\begin{array}{l}\text { Established } \\
\text { performance }\end{array}$ \\
\hline \multicolumn{3}{|l|}{ Group } \\
\hline A8 & $15(20 \%)$ & $23(14 \%)$ \\
\hline A9 & $42(55 \%)$ & $120(74 \%)$ \\
\hline A10 & $19(25 \%)$ & $20(12 \%)$ \\
\hline \multicolumn{3}{|c|}{ Midbrain DA zone ${ }^{a}$} \\
\hline Lateral & $6(8 \%)$ & $9(6 \%)$ \\
\hline Intermediate & $50(66 \%)$ & $125(76 \%)$ \\
\hline Medial & $20(26 \%)$ & $29(18 \%)$ \\
\hline Sum & $76(100 \%)$ & $163(100 \%)$ \\
\hline
\end{tabular}

"The arca of midbrain DA neurons was divided into three equal mediolateral zones.

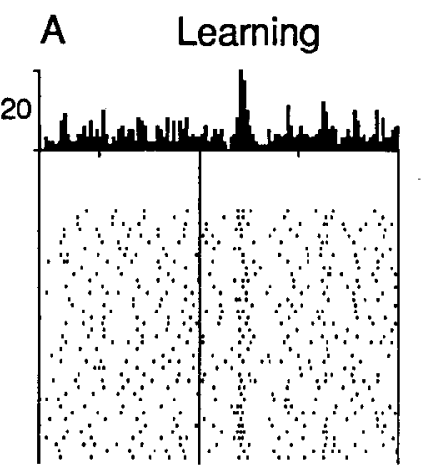

\section{Established}
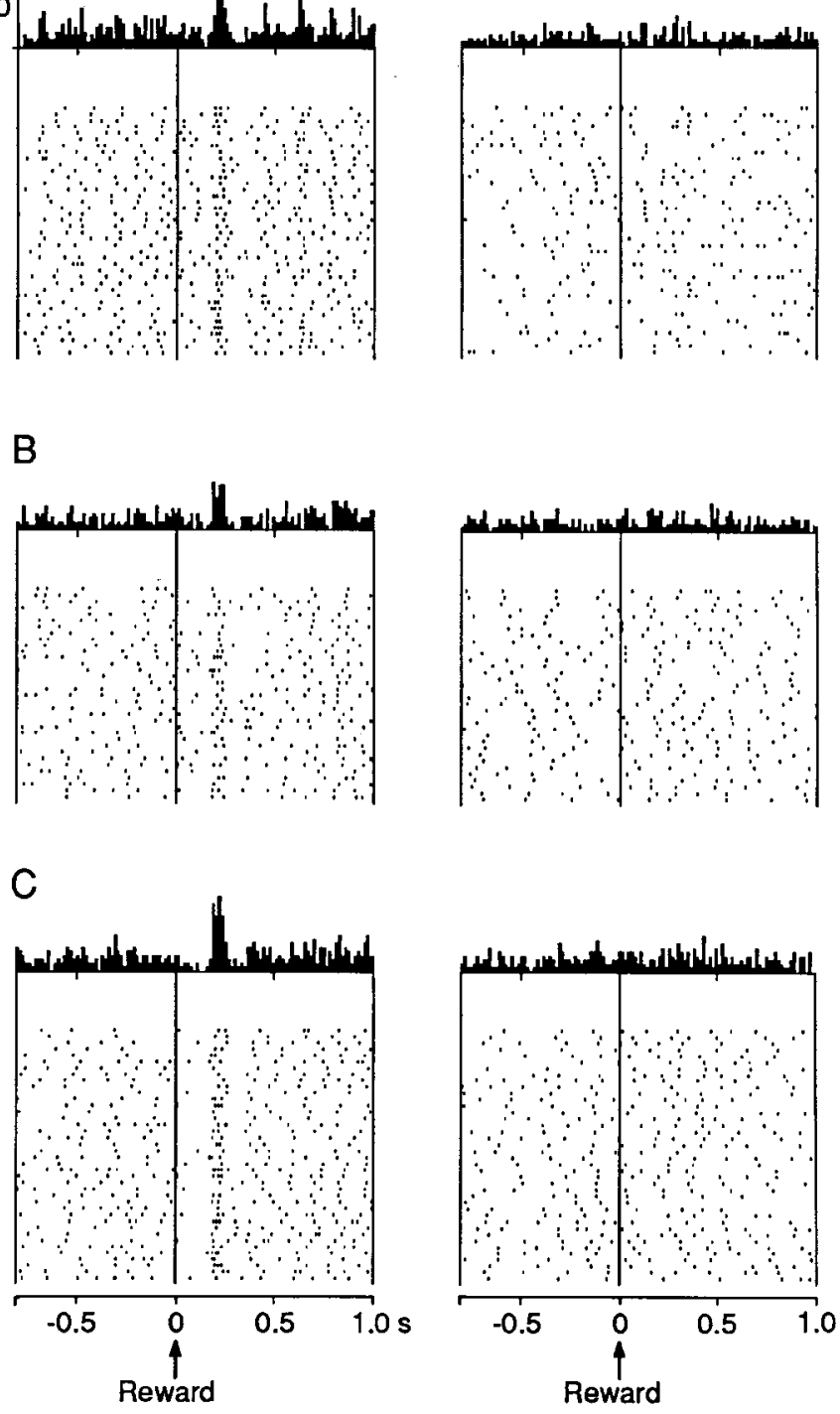

Figure 3. DA neurons respond to reward during learning but not during established task performance. $A$, Spatial choice task. $B$, Instructed spatial task. $C$, Spatial delayed rcsponse task. Neuronal activity is aligned on the electronic pulse that drives the solenoid valve delivering the reward liquid. Each panel shows the perievent time histogram and rasters of impulses from one neuron. Distances of dots to reward correspond to real-time intervals. Each line of dots shows one trial. Trials are pooled over medial and lateral levers that alternated randomly as targets for reaching during the experiment. Neurons shown on the left and right, respectively, belonged to the following groups: $A$, both $\mathrm{A} 9 ; B, \mathrm{~A} 10$ and A9; $C$, both A10. Vertical scale is 20 impulses/bin.

Table 3. Numbers of neurons responding to reward

\begin{tabular}{lrc} 
& Learning & Established \\
\hline Spatial choice task & 8 of $26(31 \%)$ & 2 of $55(4 \%)$ \\
Instructed spatial task & 5 of $22(23 \%)$ & 4 of $35(11 \%)$ \\
Spatial delayed response task & 6 of $28(21 \%)$ & 8 of $73(11 \%)$ \\
Sum & 19 of $76(25 \%)$ & 14 of $163(9 \%)$
\end{tabular}


Correct

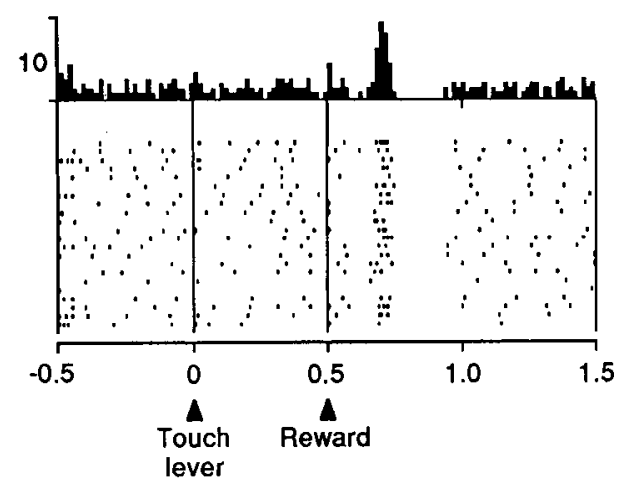

Error

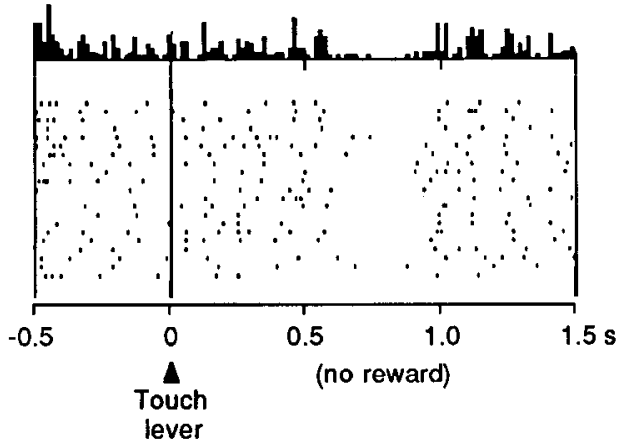

Figure 4. Activating response to reward is replaced by pure depression in erroneous trials at the time of expected reward. Left, Reward is delivered 500 msec after correct lever touch. Neuronal activity is aligned on lever touch. Right, Both reward delivery and solenoid noise are absent when the wrong lever is touched. Thus, the last stimulus precedes the depression by $>0.5 \mathrm{sec}$. Left and right panels show activity from the same A10 DA neuron during learning of the spatial delayed response task. Vertical scale is $10 \mathrm{impulses} / \mathrm{bin}$.

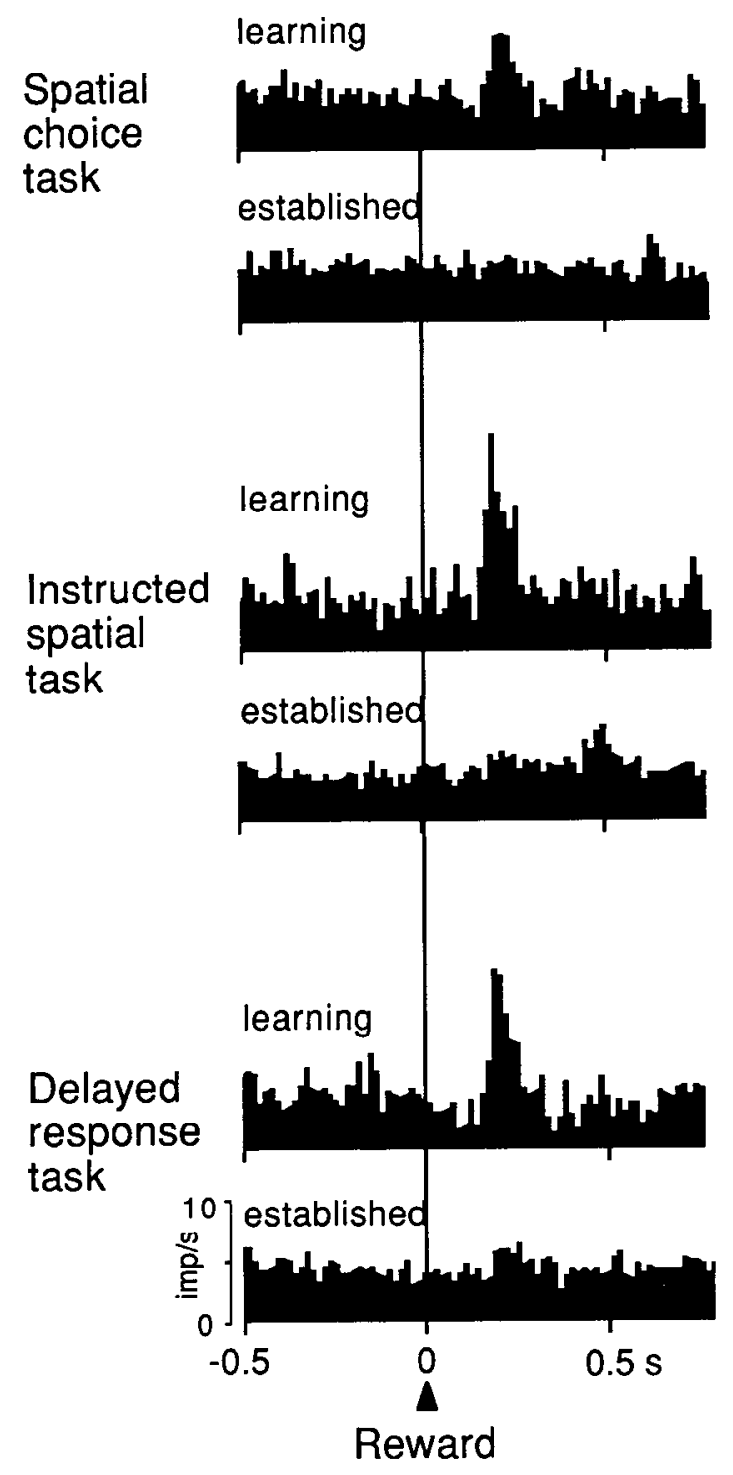

Figure 5. Population responses of DA neurons to reward during learning and established performance of the three tasks. Histograms from each neuron normalized for trial number were added and the resulting sum was divided by the number of neurons. The following data from one monkey were included for spatial choice, instructed spatial, and spatial delayed response tasks, respectively: learning, 853 trials $(n=18$ neurons), 264 trials $(n=11), 228$ trials $(n=10)$; established, 1189 trials unrewarded because the incorrect lever was touched. All of them showed depressions of activity at the time at which reward would have been delivered after correct lever touch (mean \pm SEM, $-59 \pm 9 \% ; p<0.001$ against $0 \%$, one-sample $t$ test) (Fig. 4 , right). Differences between activations in correct and depressions in incorrect trials were significant $(p<0.001$, paired $\ell$ test). Thus, the activating reward responses were replaced in error trials by pure depressions at the time of expected reward.

Reward responses in individual neurons showed mean onset latencies of $183 \pm 3 \mathrm{msec}$ and lasted for $71 \pm 3 \mathrm{msec}$. There were no significant differences in latency and duration between tasks ( $p>0.01$, one-way ANOVAs). The narrow range and variation of onset latencies and durations suggest a homogeneous temporal response character of DA neurons and allowed us to calculate the average response of all DA neurons studied in each task. This shows that reward responses during but not after learning were sufficiently strong to result in a net response of the whole population (Fig. 5). A similar difference in responsiveness to reward between learning and established performance was found when neuronal activity in the standard time window after reward was compared. Magnitudes of changes in the total neuronal population were significantly higher during learning of all three tasks, as compared to established performance (spatial choice task: $63 \pm 14 \%, n=26$, vs $21 \pm 8 \%, n$ $=55$; instructed spatial task: $106 \pm 28 \%, n=22$, vs $20 \pm 7 \%$, $n=35$; spatial delayed response task: $60 \pm 19 \%, n=28$, vs 26 $\pm 9 \%, n=73$; all $p<0.001, t$ test). Response magnitudes in the time window determined individually for each responding neuron were $242 \pm 21 \%(n=33)$. With this 3.5 -fold increase, the activity of responding DA neurons increased from about 4.5 impulses/sec baseline to an average of $15 \mathrm{impulses} / \mathrm{sec}$ during the response.

Positions of neurons responding to reward in the three tasks are shown in Figure 6. The responsiveness of DA neurons to reward during learning was significantly higher in group A10 than in groups A8 and A9, both in terms of fractions of neurons responding ( $p<0.0001$ in $\chi^{2}$ test) (Fig. 7) and in magnitudes of changes in the standard time window after reward $(p<0.01$ in one-way ANOVA). However, the responsiveness during es-

$(n=23), 803$ trials $(n=19), 1198$ trials $(n=35)$. Data were taken from all neurons recorded during each task, were only obtained from correct trials, and were pooled over trials using the medial and lateral levers. Vertical scale denotes impulses/sec. 
A

A 8.0
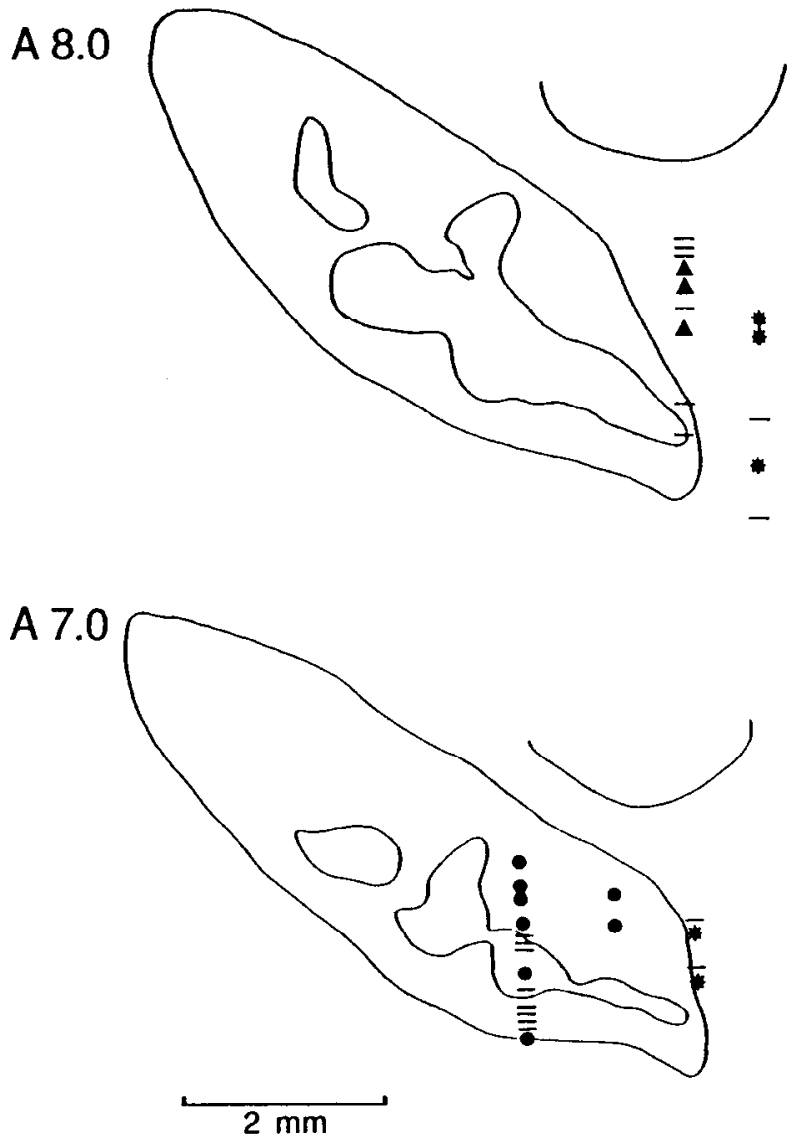

B

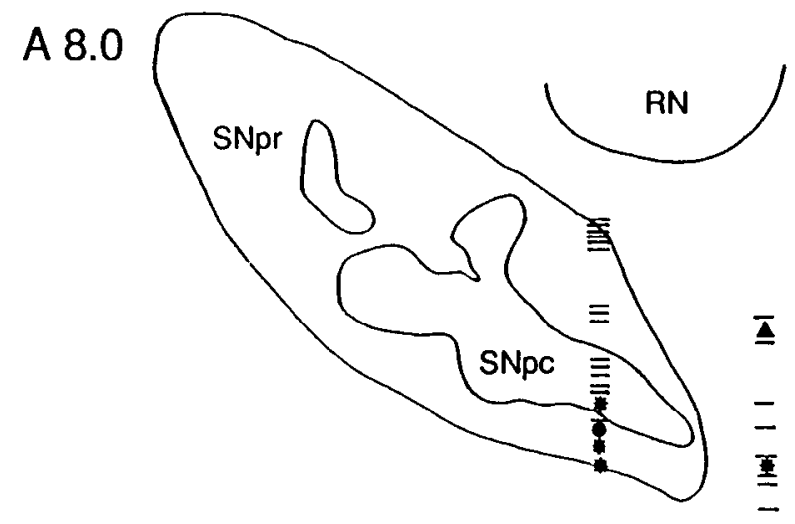

Spatial choice lask

- Instructed spatial task

- Delayed response task

- Non-responding

Figure 6. Histological reconstruction of DA neurons responding to reward in one monkey during learning $(A)$ and performance $(B)$ of the three tasks. Different symbols show positions of neurons responding in the spatial choice task (triangles), instructed spatial task (circles), and the delayed response task (asterisks), whereas dashes indicate nonresponding neurons commonly for all tasks. Coronal sections from the left hemisphere are labeled in two coronal stereotaxic planes according to the distances from the interaural line (A7.0 and A8.0). SNpr and $S N p c$, pars reticulata and pars compacta of substantia nigra; $R N$, red nucleus.

tablished task performance differed insignificantly in both of these parameters between the three groups $(p>0.1)$.

\section{Responses to conditioned stimuli}

Established task performance. The responses of DA neurons to instruction and trigger stimuli differed systematically between the three tasks (Fig. 8, Table 4). In the spatial choice task, in which the instruction and the trigger came up simultaneously, activating responses to the combined instruction-trigger stimulus occurred in 26 of 55 DA neurons (47\%) (Fig. 8, top). Only two neurons responded with depression of activity.

In the instructed spatial task, in which the trigger stimulus followed the instruction cue with a fixed interval of $1.0 \mathrm{sec}, 17$ of 35 DA neurons $(49 \%)$ responded to the instruction cue, whereas only 3 of the 35 neurons (9\%) were activated by the trigger stimulus (Fig. 8, middle). Two neurons were depressed by each stimulus. The temporal aspect of the animals' behavior in this task was mainly determined by the instruction cue. This is suggested by the saccadic reaction to the instruction and its absence to the trigger, and by some of the arm movement reaction times that were too short to be related to the trigger stimulus (Fig. 8, middle).

In the spatial delayed response task, the trigger stimulus followed instruction onset with a randomly varying interval of 2.5-3.5 sec, and the instruction cue was only shown for $1 \mathrm{sec}$. Neuronal responses occurred to both instruction and trigger stimuli and were accompanied by separate ocular reactions to each stimulus (Fig. 8, bottom). Of 73 neurons tested, 28 (38\%) showed activating responses to the instruction and $36(49 \%)$ to the trigger, 25 of them being activated by both stimuli. One neuron was depressed by the trigger. The relatedness between instruction and trigger responses is further suggested by the good correlation of magnitudes of changes in the standard time window between instruction and trigger stimuli $(r=0.8 ; p<0.01$; Spearman rank correlation test), which contrasts with the weak correlation between the instruction and reward $(r=0.2 ; p>$ 0.01) (Fig. 9).

None of the neurons showed responses that were specifically related to the medial or lateral lever being used (Fig. 10). Magnitudes of responses to the instruction or trigger stimuli in all 


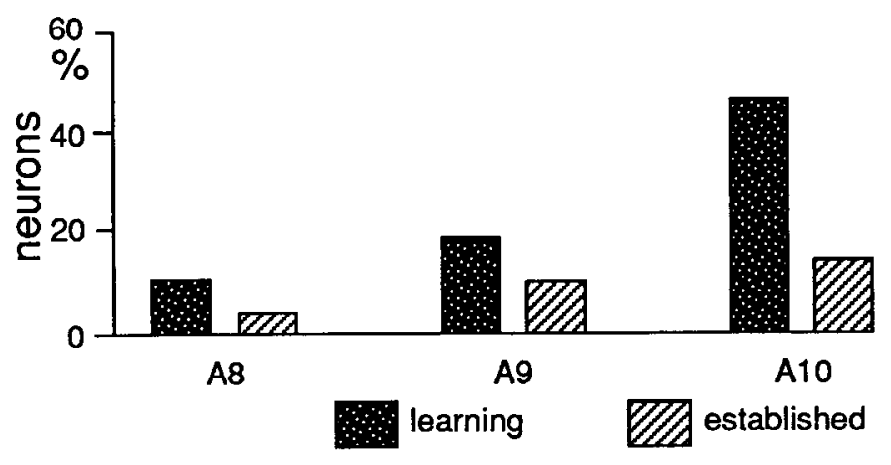

Figure 7. Distribution of DA neurons responding to reward over midbrain cell groups A8-A10 in both monkeys. Neurons from all three tasks are included. Ordinate indicates percentage of responding neurons in each cell group. The fraction of neurons responding during learning is significantly higher in group A10, as compared to groups A8 and A9 ( $p<0.0001, \chi^{2}$ test). See Table 2 for numbers of neurons tested.

neurons varied insignificantly in all three tasks between trials using the medial versus the lateral lever.

Responses to instruction and trigger stimuli in the three tasks showed mean onset latencies of 103-112 msec and 101-120 msec, respectively. There were no significant differences between tasks and stimuli ( $p>0.01$, two-way ANOVA), their common mean being $105 \pm 2$ msec. Durations of instruction responses differed insignificantly between tasks and showed a common mean of $91 \pm 3 \mathrm{msec}$ ( $p>0.01$, one-way ANOVA). Durations of trigger responses differed between tasks, being shortest in the instructed spatial task $(70 \pm 11 \mathrm{msec})$ and longest in the spatial delayed response task $(113 \pm 5 \mathrm{msec})(p<0.001$, Fisher test after one-way ANOVA). The average response of all neurons recorded in each task illustrates the strong population response to the combined instruction-trigger stimulus during established performance of the spatial choice task, and the slightly weaker responses to individual instruction and trigger stimuli in the two subsequent tasks (Fig. 11). This is also shown by the magnitudes calculated in the standard time window on all neurons recorded. Average magnitudes of changes after the instruclion were $126 \pm 25 \%, 75 \pm 17 \%$, and $77 \pm 12 \%$ in the spatial choice, instructed spatial, and spatial delayed response tasks, respectively. Comparable changes after the trigger stimulus were $4 \pm 8 \%$ and $106 \pm 15 \%$ in the instructed spatial and spatial delayed response tasks, respectively. Response magnitudes in the time window determined individually for each responding neuron in the three tasks were $277-410 \%(n=71)$ and $268-$ $410 \%(n=65)$ for instruction and trigger responses, respectively. With this four- to fivefold increase, the activity of responding DA neurons increased from about 4.5 impulses/sec baseline to an average of 17-23 impulses/sec during the response.

Learning. In the spatial choice and instructed spatial tasks, the responsiveness of DA neurons to instruction and trigger stimuli during learning was higher compared to established task

Figure 8. Responses of three DA neurons of group A9 to conditioned stimuli during task performance. Superimposed horizontal components of eye movements $(h-e o g)$ recorded simultaneously with neurons are shown above histograms and rasters of neuronal impulses and illustrate the animal's ocular reaction to stimulus presentation ( $u p=$ eyes to the right). Small vertical bars in rasters indicate movement onset (release of resting key). Trials are pooled over medial and lateral levers that
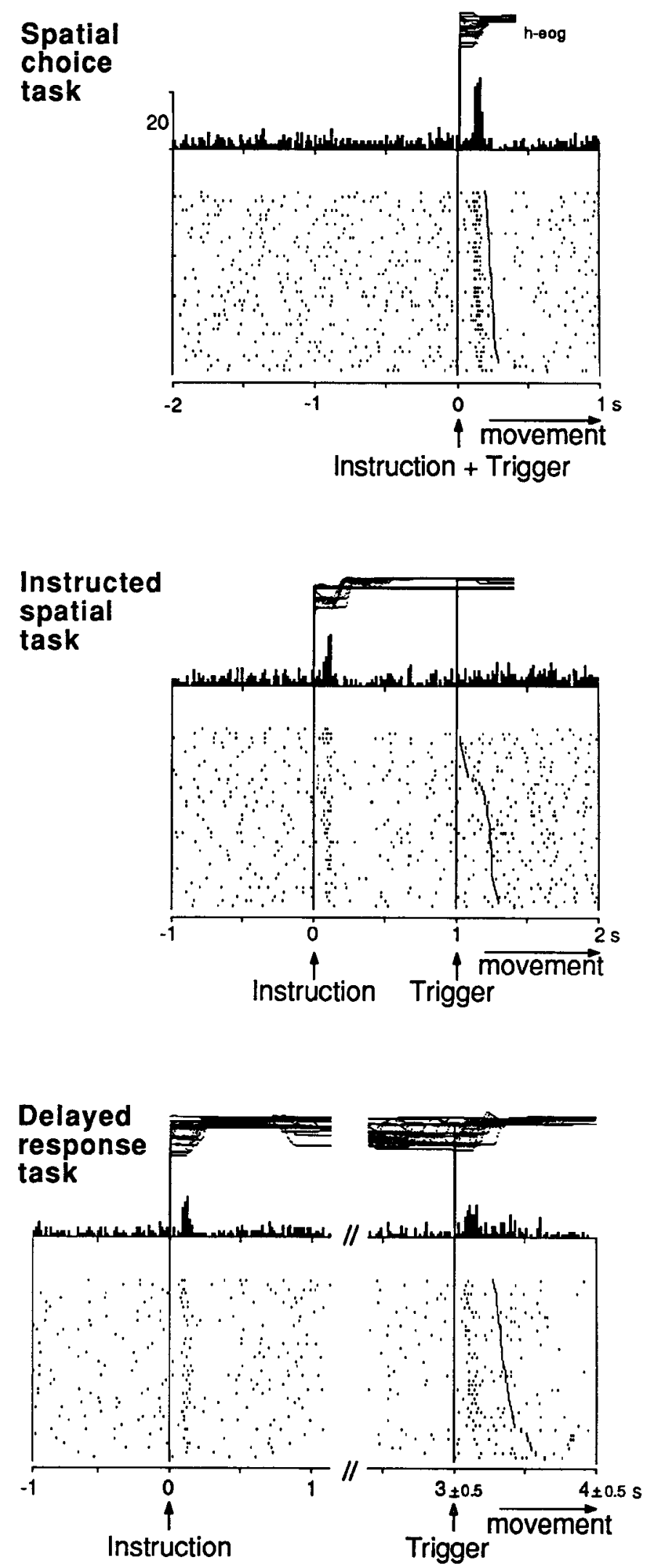

alternated randomly as targets for reaching in each block of trials. Trials were ordered off line according to reaction time (from trigger to movement onset). Because of randomly varying intervals between instruction and trigger stimuli $(3 \pm 0.5 \mathrm{sec})$, the time base in $C$ is split without maintaining the original instruction-trigger interval (rasters separately aligned to instruction and trigger stimuli). Vertical scale is 20 impulses/ bin. 
Table 4. Numbers of neurons responding to instruction and trigger stimuli

\begin{tabular}{lrl} 
& Learning & Established \\
\hline Instruction cue & & \\
$\quad$ Spatial choice task & 20 of $26(77 \%)$ & 26 of $55(47 \%)$ \\
Instructed spatial task & 16 of $22(73 \%)$ & 17 of $35(49 \%)$ \\
Spatial delayed response task & 8 of $28(29 \%)$ & 28 of $73(38 \%)$ \\
Sum & 44 of $76(58 \%)$ & 71 of $163(44 \%)$ \\
Trigger stimulus & & \\
$\quad$ Spatial choice task & 20 of $26(77 \%)$ & 26 of $55(47 \%)$ \\
Instructed spatial task & 3 of $22(14 \%)$ & 3 of $35(9 \%)$ \\
Spatial delayed response task & 6 of $28(21 \%)$ & 36 of $73(49 \%)$ \\
Sum & 29 of $76(38 \%)$ & 65 of $163(40 \%)$ \\
\hline
\end{tabular}

performance, both in terms of fractions of neurons responding and in magnitudes of changes in the standard time windows (Table 4, Fig. 12). In the spatial delayed response task, differcnces betwecn lcarning and cstablished performance varicd bctween monkeys, resulting in an overall lower responsiveness during learning.

Recording positions. Positions of neurons recorded from one monkey during the three tasks are shown in Figure 13. During learning of the three tasks, a significantly higher fraction of neurons in group A10 responded to instruction or trigger stimuli, as compared to groups A8 and A9 (both $p<0.0003, \chi^{2}$ test), although the magnitudes of changes in all neurons tested differed only insignificantly between these three areas ( $p>0.01$, oneway ANOVA) (Fig. 14). Only insignificant regional variations in both the fraction of responding neurons and the magnitudes of changes in all neurons tested were seen during established task performance, for both instruction and trigger stimuli $(p>$ 0.01 in both $\chi^{2}$ and one-way ANOVA tests).

\section{Discussion}

These data show that DA neurons respond to those stimuli that are crucial for learning and performing a behavioral task. In the spatial tasks studied presently, the stimuli effective for activating DA neurons were (1) the delivery of primary reward during learning, which alerts the animal, serves as reinforcer, and indicates correct performance in each task; (2) the instruction cue as first stimulus in each trial, which also provides the necessary spatial information for task performance; (3) the trigger stimulus constituting a conditioned incentive stimulus that predicts reward and elicits an ocular reaction and an arm reaching movement. Most responding neurons were activated by more than one stimulus, indicating that the responses were not selective for the particular behavioral processes associated with the stimuli, such as reinforcement, spatial information, or initiation of movement. Rather, the responses appear to be related to the significance of the stimuli in the particular behavioral situation.

\section{Reward responses during learning}

Primary rewards are food objects or fluids that are approached by subjects through innate or instinctive behavior or are learned very early during ontogenesis. Primary rewards may serve to establish and sustain learned behavior, in which case they are called primary reinforcers. A fraction of DA neurons responded to the delivery of primary liquid reward during the learning
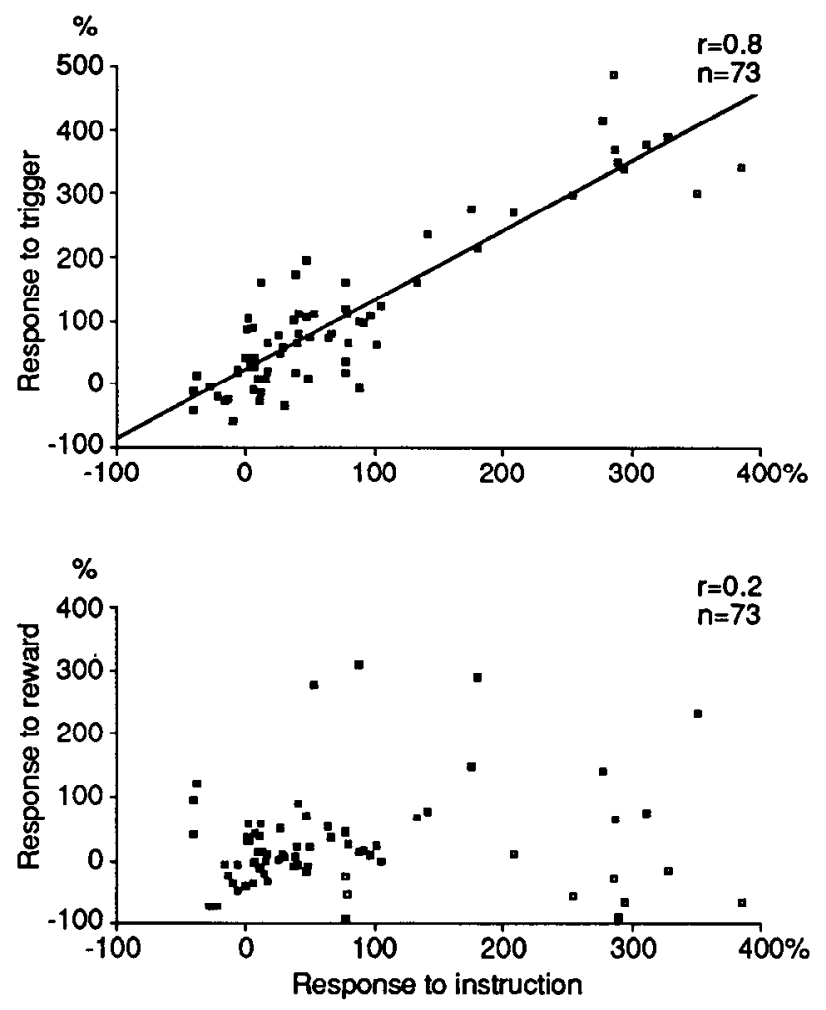

Figure 9. Correlations of magnitudes of neuronal changes between different stimuli. Spearman's rank correlation shows a high coefficient between instruction and trigger stimuli $(r=0.8 ;$ top $)$, but a low coefficient between instruction and reward $(r=0.2 ;$ bottom $)$. Magnitudes of changes in respective standard time windows are shown from all neurons recorded during established task performance in the spatial delayed response task in both monkeys. Mean changes were $77 \pm 12 \%, 106 \pm$ $15 \%$, and $26 \pm 9 \%$ after instruction, trigger, and reward, respectively.

phase of each of the three successive tasks. The responses were sufficiently frequent and strong to result in a net increase of activity in the whole population of neurons recorded during learning. By contrast, the small fraction of neurons responding to reward during established task performance and their low response magnitude failed to result in such a net population response, suggesting that these responses were little more than random fluctuations in responsiveness among individual neurons. Reward responses during learning occurred more frequently but not exclusively in group A10, suggesting a moderate gradient of responsiveness over groups A8-A10. This might induce a predominant reward-related dopaminergic influence on the postsynaptic structures innervated by A 10 neurons, such as the ventral striatum, amygdala, and certain areas of frontal cortex (Szabo, 1980; Porrino and Goldman-Rakic, 1982).

The predominant occurrence of reward responses during the learning phase of each task corresponds to the restriction of similar reward rcsponscs to the learning phase of a simple reaction time task (Ljungberg et al., 1992). However, the fraction of responding neurons was only about one-half of that seen during learning of the reaction time task. The present results were obtained in animals trained before in the reaction time task using the same behavioral apparatus (resting key, target lever, mode of reward delivery). It is conceivable that the delivery of reward constitutes a more alerting stimulus for an animal during learning the first task, as compared to learning 

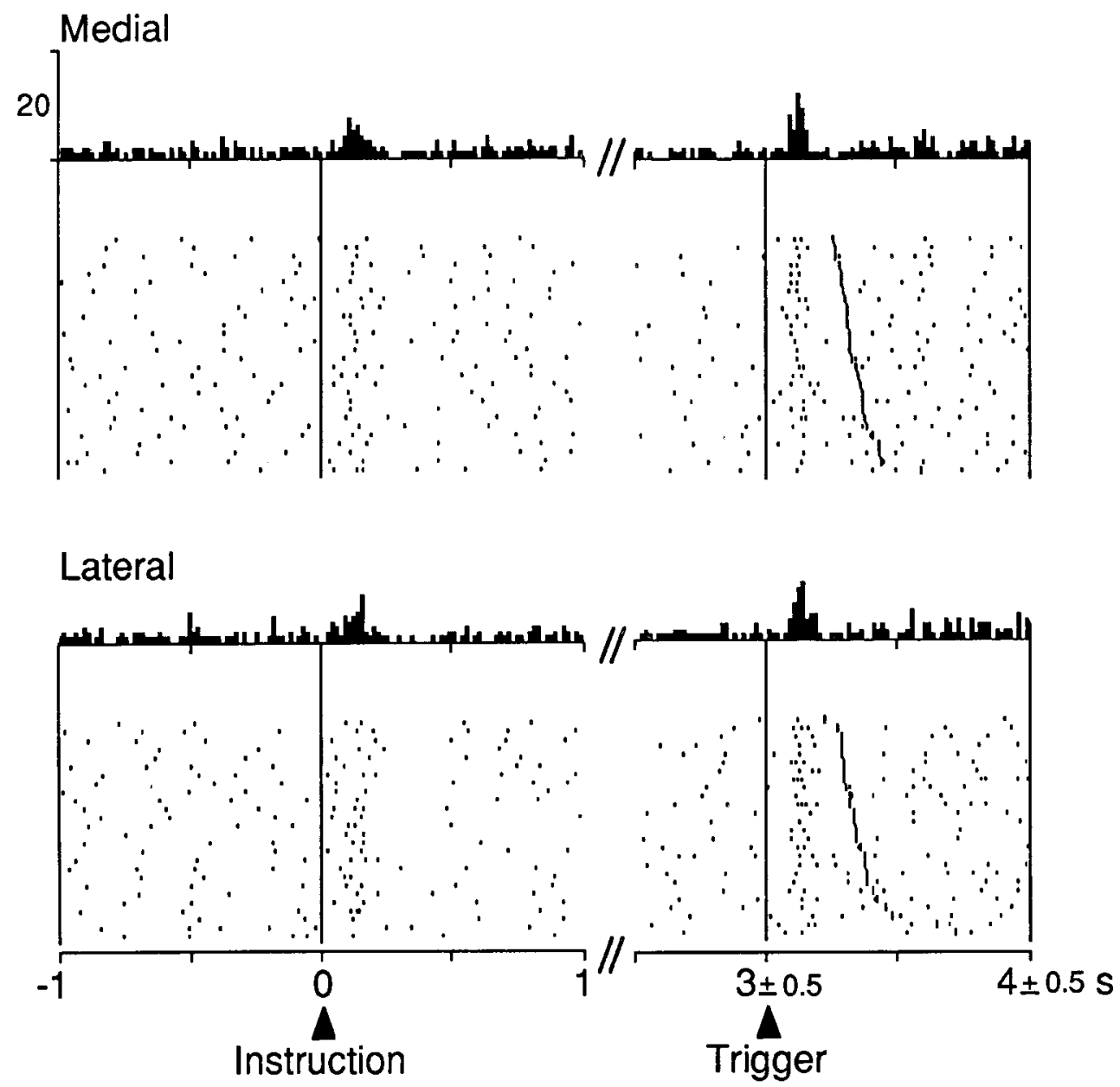

Figure 10. Responses to instruction and trigger stimuli are independent of movement target. Data are from one A9 DA neuron during established performance in the delayed response task. Trials using medial and lateral levers alternated randomly during each block and were separated for analysis and ordered according to reaction time (from trigger to movement onset). Small vertical lines in rasters indicate movement onset (release of resting key). Because of randomly varying intervals between instruction and trigger stimuli $(3 \pm 0.5$ sec), the time base is split without maintaining the original instructiontrigger interval. Vertical scale is impulses/bin.
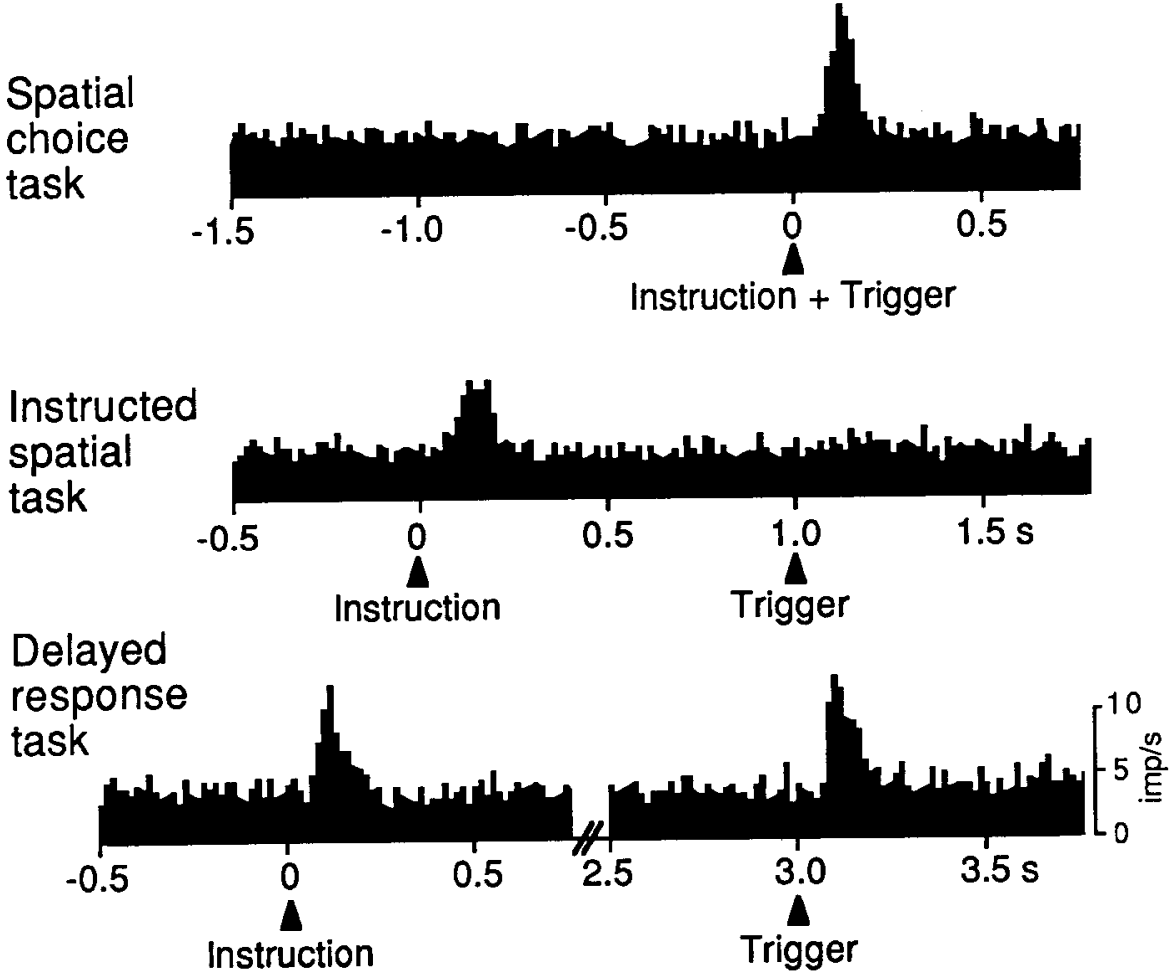

Figure 11. Population responses of DA neurons to instruction and trigger stimuli during established performance of the three tasks. Because of varying intervals, histograms were separately referenced to instruction onset and trigger stimulus for the spatial delayed response task. The following data wcrc included from one monkey for spatial choice, instructed spatial, and spatial delayed response tasks, respectively: 1189 trials ( $n=23$ neurons), 803 trials $(n=19), 1198$ trials $(n=35)$. Data were taken from all neurons recorded during each task and pooled over correctly performed trials using the medial and lateral levers. Vertical scale is impulses/ bin. 


\section{Instruction}

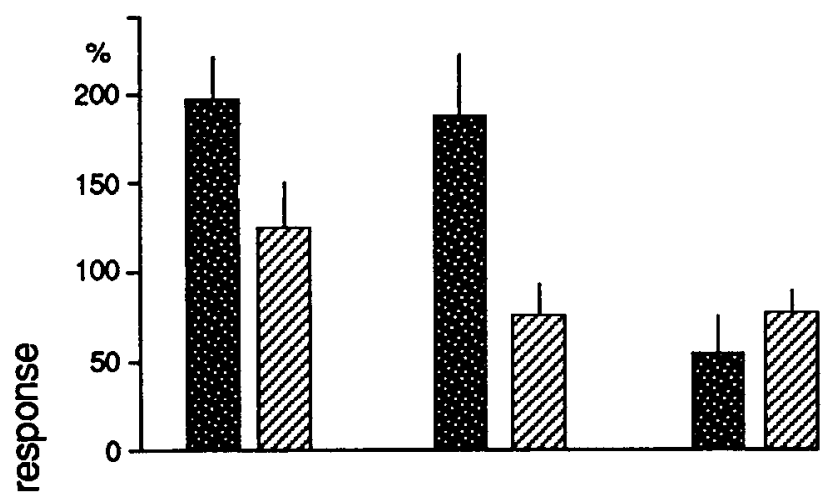

Trigger

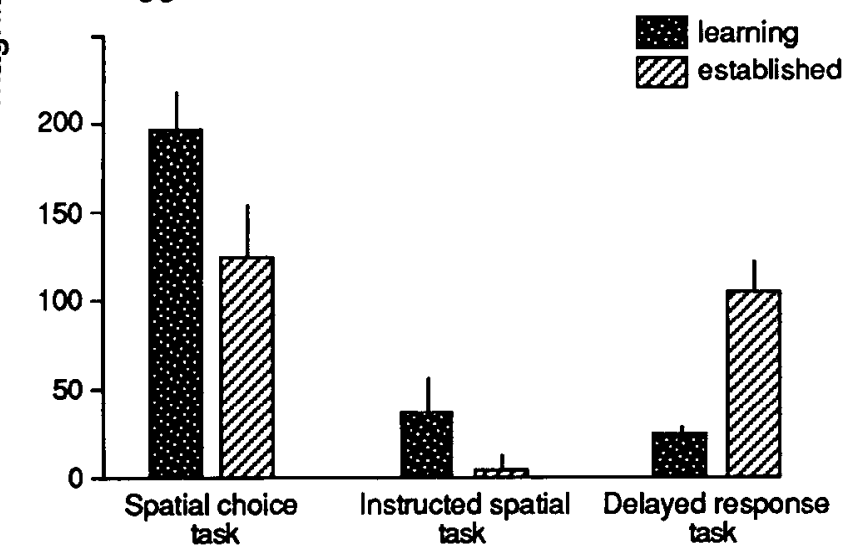

Figure 12. Magnitudes of changes after instruction and trigger stimuli in all neurons tested. The standard time window procedure was employed on all DA neurons tested in both monkeys during learning and established performance of each task, respectively. Differences in magnitudes of changes between learning and established performance were significant for instruction responses in the instructed spatial task $(p<$ $0.001, t$ test), whereas all other comparisons failed to reach this level. See Table 3 for numbers of neurons tested.

\section{A 7.0}

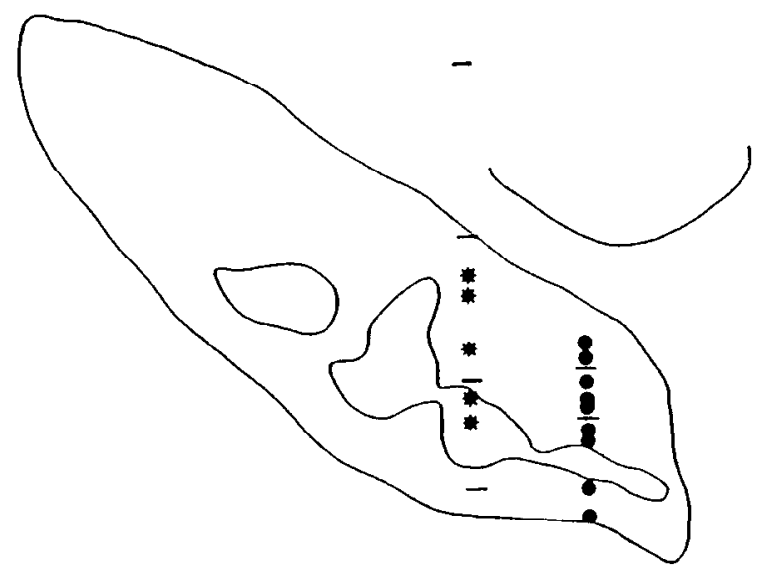

$2 \mathrm{~mm}$

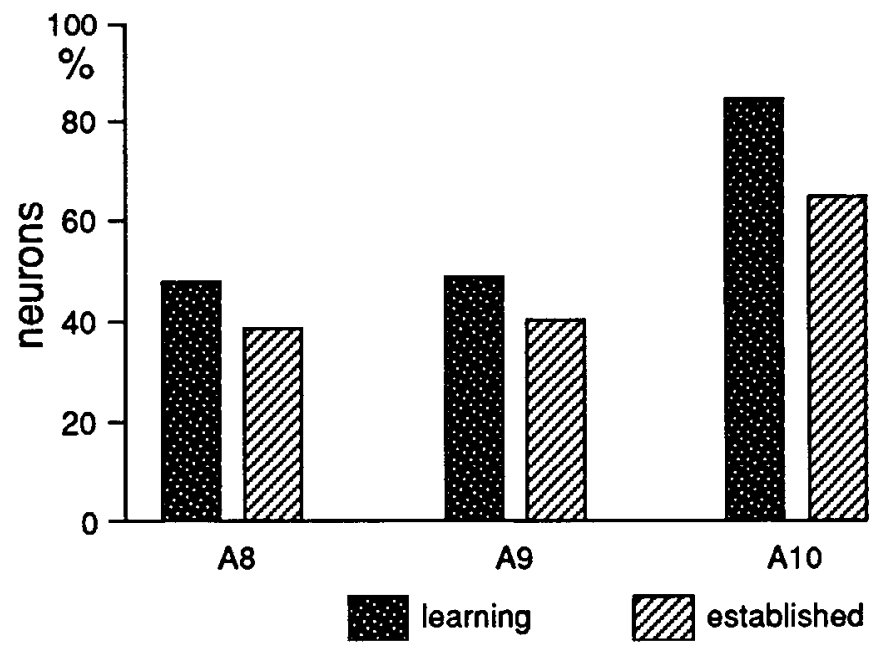

Figure 14. Distribution of DA neurons responding to the instruction cue over midbrain cell groups A8-A 10 in both monkeys. Neurons from all three tasks are included. Ordinate indicates percentage of responding neurons in each cell group. The fraction of neurons responding during learning is significantly higher in group A10, as compared to groups A8 and A9 $\left(p<0.0001, \chi^{2}\right.$ test). See Table 2 for numbers of neurons tested.

the following three tasks in which the arrival of reward and its meaning for task learning are already somewhat familiar.

The fraction of neurons presently responding to reward was also lower than that observed during learning of a delayed alternation task (Ljungberg et al., 1991). This may be due to the double role of reward in that task, serving as both reinforcer and indirect indicator of the next movement target. In agreement with this reasoning, reward responses persisted during established performance of the delayed alternation task, even after overtraining, whereas the present reward responses were largely restricted to the learning phase.

Several arguments suggest that the observed reward responses did not constitute startle responses to stimuli occurring unexpectedly to the animal. Each animal was very familiar with a drop of liquid being delivered at the spout in front of its mouth

\section{A 8.0}

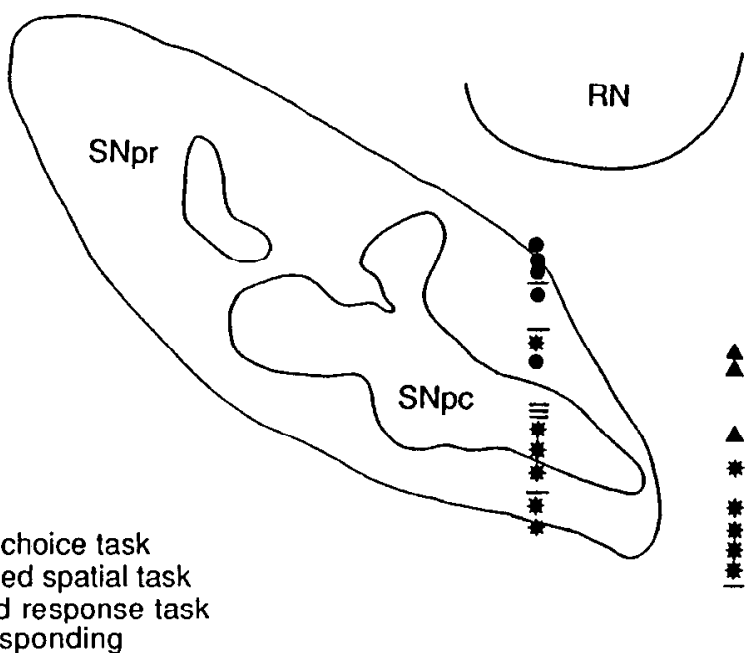

A Spatial choice task

- Instructed spatial task

- Delayed response task

- Non-responding

Figure 13. Recording positions of DA neurons with instruction responses during established task performance in one monkey. Positions on two coronal levels are marked separately for each task, whereas nonresponding neurons are commonly labeled by a dash. $S N p r$ and $S N p c$, pars reticulata and pars compacta of substantia nigra; $R N$, red nucleus. 
through its prior learning, performance, and overtraining in the reaction time task with several tens of thousands of trials. Reward responses were largely absent during established task performance and regularly came back with similar frequencies when reward contingencies were changed when advancing from one task to the next one. None of the DA neurons showed response habituation over several tens of consecutive trials, a result that was also seen with all other stimuli effective for activating DA neurons in these tasks. The relation to the delivery of liquid and the lack of relation to stimuli associated with this event are suggested by the effects of closing the liquid tube, as tested in the delayed alternation task (Ljungberg et al., 1991). This manipulation left the noise of the solenoid liquid valve present while blocking liquid flow. DA neurons responding to reward failed to be activated in this situation. A similar specificity for reward was seen before when DA neurons responded to the touch of food reward but failed to be activated by inedible objects (Romo and Schultz, 1990).

An interesting finding was the reproducible depression of activity in error trials occurring in all DA neurons at the precisely the moment when reward would have been delivered in correct trials. The depression did not occur in close temporal relation to a stimulus or a particular behavioral event but appeared as a sharp decrease in activity more than $500 \mathrm{msec}$ after the wrong lever was touched. Reward was normally given at this fixed delay of $500 \mathrm{msec}$ after lever touch throughout the experiment with each animal. It had already been employed for several tens of thousands of trials during learning, established performance, and overtraining of the preceding reaction time task. This suggests that the depression was not a response to the preceding event but reflects a state of expectation of reward at the time of its usual delivery. This would imply that the input to DA neurons had access to centrally stored information concerning the expected time of reward delivery. A similar depression of activity in DA neurons was seen in error trials and in trials with a closed liquid tube during a delayed alternation task (Ljungberg et al., 1991) and when food morsels were replaced by hidden inedible objects (Romo and Schultz, 1990). The visual inspection of rasters and histograms revealed that depressions occurred in many neurons even in the presence of reward responses after correct performance, such that the activating response was added on top of the depressed activity. These depressions were lost together with the activating reward responses after established task performance. This suggests the convergence of two different inputs to DA neurons, one activating input directly driven by the delivery of reward, and one depressant input carrying information about the expected time of arrival of reward but without indicating whether reward was actually delivered.

In conclusion, the restricted occurrence of reward responses during the learning phase and not during established task performance suggests a relationship to the acquisition of new task contingencies. The low responsiveness during established task performance rules out a relationship to general task reinforcement. The fact that many of the same DA neurons also respond to conditioned stimuli important for task performance suggests that reward belongs to a larger class of stimuli effective for activating DA neurons. These stimuli apparently have a particularly alerting and attention-grabbing function for the animal. The particular motivational value of the reception of reward during learning makes this signal a salient stimulus that is very important for the animal when trying to find the new task con- tingencies. This characteristic appears to determine is capacity to drive DA neurons. Thus, stimuli of prime motivational significance form an important albeit not exclusive class of salient stimuli driving DA neurons. The activity of DA neurons would not selectively transmit information about the reception of primary reward but indicates that a particularly important stimulus occurred in the environment of the subject.

\section{Responses to conditioned stimuli}

Whereas reward responses were largely restricted to the learning phase and disappeared with established task performance, responses to the instruction and trigger stimuli occurred throughout both learning and established performance, albeit at varying frequencies. This is probably due to the fact that their behavioral significance was partly known to the animals from the preceding reaction time task. DA neurons failed to respond to known stimuli without any specific meaning for the animal's behavior and only began to respond when the significance of the stimulus became known through learning of the first task (Ljungberg et al., 1992). The change of behavioral significance of familiar conditioned stimuli induced by the modified reward contingencies during the different steps of learning obviously has another impact on DA neurons than the first association of behavioral significance to a familiar but neutral stimulus. The variations of neuronal responsiveness between learning and established performance in each task and between the three tasks should then be related to the changes in behavioral significance of the stimuli during these periods.

Spatial choice task. Animals were familiar with the trigger stimulus from the previous reaction time task (Ljungberg et al., 1992). In the present task, an additional instruction cue was illuminated simultaneously with the trigger stimulus to indicate which of the two levers had to be touched. This visually guided reaching to different targets differed from the single target reaching of the preceding reaction time task and introduced a spatial choice as a first step toward learning the spatial delayed response task. The animal in addition needed to choose between two possible targets in the spatial choice task and thus pay more attention to the conditioned stimuli. Whereas neuronal responses to the trigger stimulus were very low after overtraining of the preceding reaction time task, they reappeared when a spatial choice was required in the present tasks. The increased responsiveness should mainly be due to the increased alerting and attention-grabbing function of the reward-predicting trigger stimulus induced by the changed behavioral situation. We have suggested that responses to triggering stimuli were due to the reward-predicting nature of this conditioned stimulus (Schultz and Romo, 1990; Ljungberg et al., 1992). The present responses to the combined instruction-trigger stimulus are probably not due to the higher sensory impact of two simultaneous visual stimuli, because responses declined again when task performance was established. They should also not be related to the specific spatial information contained in the instruction because they lacked spatial specificity.

Instructed spatial task. As the second step toward learning the spatial delayed response task, the instruction cue indicating the target of reaching was separated by a fixed period of $1 \mathrm{sec}$ from the following trigger stimulus, which determined the moment of reaching. The animal learned to withhold reaching after the instruction cue and wait until the trigger stimulus occurred. This change in the behavioral situation had two effects on DA neurons. Neuronal responsiveness increased again during learn- 
ing of this task, and the responses occurred virtually exclusively to the instruction cue and not to the trigger stimulus. Animals reacted with a saccadic eye movement toward the position of the instruction-lever compound and fixated this target until the reaching movement occurred, whereas the trigger stimulus failed to elicit an cye movement. Also, the arm movement began in several trials too soon after the trigger to be elicited by this stimulus. This suggests that the instruction, besides indicating the spatial position, served to trigger a delayed arm movement and that the trigger light had largely lost its unique triggering function and merely served as a temporal reference for the delayed movement. Thus, the neuronal responses to the instruction cue and their absence after the trigger stimulus should be explained by a transfer of the alerting, attention-grabbing, and reward-predicting characteristics from the trigger stimulus to the instruction cue.

Spatial delayed response task. The last step in learning this task involved two modifications. The instruction-trigger interval was randomized and prolonged to $2.5-3.5 \mathrm{sec}$. A delay was introduced between instruction offset and trigger onset during which task specific information was absent and the animal had to memorize the spatial position of the target lever indicated by the instruction. The response of DA neurons became immediately separated into a response to the instruction and, in the same trial, a response to the trigger stimulus with very similar temporal characteristics. This resulted in a lowering of responsiveness to each stimulus. Responses increased with established task performance, and a slightly higher fraction of neurons responded to the trigger as compared to the instruction cue. The study of eye and arm movements indicated that the two signals had attained different characteristics. The instruction cue was the first stimulus in each trial and also provided important spatial information for correct task performance. It elicited a saccadic eye movement. The trigger stimulus was again a distinctive stimulus that elicited a separate eye movement and the reaching movement toward the target. As principal reward predictor, the trigger stimulus was the main incentive stimulus of the task. However, the instruction cue through its close temporal association with the trigger stimulus and reward may not have been completely devoid of incentive properties.

It is presently unclear whether the stimulus properties of the instruction cue are due to its general role in the delayed response task or are derived from the particular training procedure employed. It also remains to be shown to what extent instruction responses would be reduced with overtraining. In a previous experiment, instruction responses occurred in an asymmetrically reinforced delayed go-no-go task only when the task was occasionally employed, whereas they disappeared with frequent use (Schultz and Romo, 1990). A systematic investigation of responses to instructions in different behavioral contexts would be an interesting way to study the relationship of DA neurons to stimuli not associated with the immediate obtainment of reward.

\section{Role of DA neurons in delay tasks}

Previous studies investigating the role of DA in animal cognition found that local DA depletions or local administration of DA receptor antagonists into the prefrontal cortex of monkeys result in impaired performance of spatial delayed response tasks (Brozoski et al., 1979; Sawaguchi and Goldman-Rakic, 1991). During the performance of delayed response tasks similar to the one used presently, prefrontal neurons respond phasically to spatial instruction cues that are being memorized, and to trigger stimuli that induce the recall of stored information and elicit the behavioral response (Fuster, 1973; Kubota et al., 1974; Niki, 1974). Prefrontal ncurons also show sustained activity during the instruction-trigger delay, which may reflect processing related to representational task components, such as working memory, expectation of the trigger stimulus, and preparation of the behavioral response. Similar activity is found in striatal neurons during the performance of delay tasks (Alexander, 1987; Schultz and Romo, 1988), including the same spatial delayed response task in the two monkeys used presently (Apicella et al., 1992). DA receptor antagonists reduce and DA itself increases both the phasic responses and the sustained delay activity of prefrontal neurons when applied iontophoretically (Sawaguchi et al., 1990a,b). These combined findings would explain the deficits in delayed response performance after impaired DA transmission and suggest that prefrontal neuronal activity is directly responsible for the performance of delayed response tasks. By contrast, DA neurons failed to show sustained activations during the delay, suggesting that they do not encode the abovementioned representational task components. The phasic responses to instruction and trigger stimuli were not specific for the spatial component of task performance but were apparently related to the salience of these stimuli. Thus, DA neurons appear to enable prefrontal neuronal activity related to representational task components underlying delayed response performance without specifically encoding these components. The mechanisms of this enabling function are presently unknown. It is possible that sustained neuronal activity is facilitated by the action of ionic channels with slow time constants in cortical and striatal neurons (Stafstrom et al., 1982; Calabresi et al., 1987; Kawaguchi et al., 1989; Pennartz et al., 1991) and is built up by cortico-basal ganglia-cortical loops (Romo and Schultz, in press). It would be very interesting to see whether DA may have a specific influence on the time constants of these channels and thus have a regulatory effect on the passage of neuronal activity in these loops.

\section{References}

Alexander GE (1987) Selective neuronal discharge in monkey putamen reflects intended direction of planned limb movements. Exp Brain Res 67:623-634.

Apicella P. Scarnati E, Ljungberg T, Schultz W (1992) Neuronal activity in monkey striatum related to the expectation of predictable environmental events. J Neurophysiol 68:945-960.

Brozoski TJ, Brown RM, Rosvold HE, Goldman PS (1979) Cognitive deficit caused by regional depletion of dopamine in prefrontal cortex of rhesus monkey. Science 205:929-932.

Calabresi P. Mercuri N, Stanzione P. Stefani A, Bernardi G (1987) Intracellular studies on the dopamine-induced firing inhibition of neostriatal neurons in vitro: evidence for D1 receptor involvement. Neuroscience 20:757-771.

Cools AR, Van den Bercken JHL, Horstink WI, Van Spaendonck KPM, Berger HJC (1984) Cognitive and motor shifting aptitude disorder in Parkinson's disease. J Neurol Neurosurg Psychiatry 47:443-453.

Felten DL, Sladeck JR (1983) Monoamine distribution in primate brain. V. Monoaminergic nuclei: anatomy, pathways and local organisation. Brain Res Bull 10:171-284.

Fuster JM (1973) Unit activity of prefrontal cortex during delayedresponse performance: neuronal correlates of transient memory. J Neurophysiol 36:61-78.

Hellweg FC, Schultz W, Creutzfeldt OD (1977) Extracellular and intracellular recordings from cat's cortical whisker projection area: thalamocortical response transformation. J Neurophysiol 40:462-479.

Kawaguchi Y, Wilson CJ, Emson PC (1989) Intracellular recording of identified neostriatal patch and matrix spiny cells in a slice preparation preserving cortical inputs. J Neurophysiol 62:1052-1068. 
Kubota K, Iwamoto T, Suzuki H (1974) Visuokinetic activities of primate prefrontal neurons during delayed-response performance. $\mathrm{J}$ Neurophysiol 37:1197-1212.

Ljungberg T, Apicella P, Schultz W (1991) Responses of monkcy midbrain dopamine neurons during delayed alternation performance. Brain Res 586:337-341.

Ljungberg T, Apicella P, Schultz W (1992) Responses of monkey dopamine neurons during learning of behavioral reactions. J Neurophysiol 67:145-163.

Miller JD, Sanghera MK, German DC (1981) Mesencephalic dopaminergic unit activity in the behaviorally conditioned rat. Life Sci 29:1255-1263.

Niki H (1974) Differential activity of prefrontal units during right and left delayed response trials. Brain Res 70:346-349.

Pennartz CMA, Boeijinga PH, Kitai ST, Lopes da Silva FH (1991) Contribution of NMDA receptors to postsynaptic potentials and pairedpulse facilitation in identified neurons of the rat nucleus accumbens in vitro. Exp Brain Res 86:190-198.

Porrino LJ, Goldman-Rakic PS (1982) Brainstem innervation of prefrontal and anterior cingulate cortex in the rhesus monkey revealed by retrograde transport of HRP. J Comp Neurol 205:63-76.

Romo R, Schultz W (1990) Dopamine neurons of the monkey midbrain: contingencies of responses to active touch during self-initiated arm movements. J Neurophysiol 63:592-606.

Romo R, Schultz W (in press) Role of primate basal ganglia and frontal cortex in the internal generation of movements: neuronal activity in the supplementary motor area. Exp Brain Res, in press.

Saint-Cyr JA, Taylor AE, Lang AE (1988) Procedural learning and neostriatal function in man. Brain 111:941-959.

Sawaguchi T, Goldman-Rakic PS (1991) D1 Dopamine receptors in prefrontal cortex: involvement in working memory. Science 251:947950.
Sawaguchi T, Matsumura M, Kubota K (1990a) Catecholaminergic effects on neuronal activity related to a delayed response task in monkey prefrontal cortex. J Neurophysiol 63:1385-1400.

Sawaguchi T, Matsumura M, Kubota K (1990b) Effects of dopamine antagonists on neuronal activity related to a delayed response task in monkey prefrontal cortex. J Neurophysiol 63:1401-1412.

Schneider JS, Kovelowski CJ (1990) Chronic exposure to low doses of MPTP. I. Cognitive deficits in motor asymptomatic monkeys. Brain Res 519:122-128.

Schultz W (1986) Responses of midbrain dopamine neurons to behavioral trigger stimuli in the monkey. J Neurophysiol 56:1439-1462.

Schultz W, Romo R (1987) Responses of nigrostriatal dopamine neurons to high intensity somatosensory stimulation in the anesthetized monkey. J Neurophysiol 57:201-217.

Schultz W, Romo R (1988) Neuronal activity in the monkey striatum during the initiation of movements. Exp Brain Res 71:431-436.

Schultz W, Romo R (1990) Dopamine neurons of the monkey midbrain: contingencies of responses to stimuli eliciling immediate behavioral reactions. J Neurophysiol 63:607-624.

Schultz W, Ljungberg T, Apicella P (1991) Activity of dopamine neurons.in monkeys learning and performing cognitive tasks. Soc Neurosci Abstr 17:1218.

Simon H, Scatton B, LeMoal M (1980) Dopaminergic A10 neurons are involved in cognitive functions. Nature 286:150-151.

Stafstrom CE, Schwindt PC, Crill WE (1982) Negative slope conductance due to a persistent subthreshold sodium current in cat neocortical neurons in vitro. Brain Res 236:221-226.

Steinfels GF, Heym J, Strecker RE, Jacobs BL (1983) Behavioral correlates of dopaminergic unit activity in freely moving cats. Brain Res 258:217-228.

Szabo J (1980) Organization of the ascending striatal afferents in monkeys. J Comp Neurol 189:307-321. 